The Astrophysical Journal, 328:L35-L39, 1988 May 15

(C) 1988. The American Astronomical Society. All rights reserved. Printed in U.S.A.

\title{
WARM ULTRALUMINOUS GALAXIES IN THE IRAS SURVEY: THE TRANSITION FROM GALAXY TO QUASAR?
}

\author{
D. B. Sanders, B. T. Solfer, J. H. Elias, G. Neugebauer, and K. Matthews \\ Palomar Observatory, California Institute of Technology \\ Received 1987 December 29; accepted 1988 February 12
}

\begin{abstract}
Of 90 extragalactic objects chosen from the IRAS catalog on the basis of brightness at $60 \mu \mathrm{m}$ and "warm" infrared color $\left[f_{v}(25 \mu \mathrm{m}) / f_{v}(60 \mu \mathrm{m})>0.2\right], 12$ have luminosities characteristic of quasars. These 12 ultraluminous objects have Seyfert spectra, and nine of them show morphological evidence for recent collisions or mergers - they plausibly represent a transition stage between cooler ultraluminous infrared galaxies and optical quasars.

Subject headings: galaxies: general — infrared: general — infrared: sources - quasars
\end{abstract}

\section{INTRODUCTION}

A recent study by Sanders et al. (1988, hereafter Paper I) of the most luminous infrared galaxies in the IRAS Bright Galaxy Survey (BGS) (Soifer et al. 1987, hereafter Paper II) suggested an evolutionary connection between ultraluminous infrared galaxies having bolometric luminosities greater than $10^{12} L_{\odot}$ and quasars. Specifically, it was suggested from optical and near-infrared observations that the ultraluminous infrared objects were dust-enshrouded quasars triggered by the strong interaction/merger of two gas-rich spiral galaxies.

To discover objects with properties intermediate between those of the ultraluminous BGS objects and optical quasars, we have made use of the fact that all of the ultraluminous BGS objects have relatively "warm" infrared colors in order to select high-luminosity objects at lower observed flux levels in the IRAS data. It was noted that those galaxies that had the warmest $25 \mu \mathrm{m} / 60 \mu \mathrm{m}$ color temperatures, e.g., Mrk 231, displayed the closest appearance to "classical" quasars, i.e., broad emission lines, dominant optical nucleus, etc. This suggests that the bridging of the "gap" between the ultraluminous BGS objects and the quasars might be found in the extragalactic objects selected to be "warmer" than those selected by a strictly $60 \mu \mathrm{m}$ flux density criterion such as used for the BGS. This approach was used previously by, e.g., de Grijp, Miley, and Lub (1987) and Low et al. (1988) to identify active galaxies in the IRAS database.

\section{THE SAMPLE}

The warm galaxies were chosen from the IRAS Point Source Catalog (PSC) on the basis of the following criteria: wellconfirmed detections at both $25 \mu \mathrm{m}$ and $60 \mu \mathrm{m}$, declination $\delta>-30^{\circ}$, galactic latitude $|b|>30^{\circ}$, and $f_{v}(60 \mu \mathrm{m})>1.5 \mathrm{Jy}$. In addition, the color criterion was imposed that $0.2<$ $f_{v}(25 \mu \mathrm{m}) / f_{v}(60 \mu \mathrm{m})<2.0$. The area constraints are nearly identical to those of the BGS and were imposed to minimize the contamination of the sample with Galactic sources while maximizing the likelihood of previous observations of the sample objects. The $60 \mu \mathrm{m}$ flux density limit was chosen to ensure the objects be reasonably bright, while the flux ratio criterion was based on the observed colors of the BGS with the intent of preferentially selecting objects warmer than commonly found in the BGS. There was a specific intent to establish some overlap between the two samples so the lower limit of the 25 $\mu \mathrm{m} / 60 \mu \mathrm{m}$ flux ratio was chosen to accept the warmest galaxies in the BGS, while remaining significantly above the mean value of $f_{v}(25 \mu \mathrm{m}) / f_{v}(60 \mu \mathrm{m}) \approx 0.1$ of galaxies in the BGS which were selected strictly at $60 \mu \mathrm{m}$.

After elimination of clearly Galactic objects, the total sample was 90 "warm" extragalactic objects in nearly the same survey area as the BGS. The flux limit of the PSC is $\sim 0.6 \mathrm{Jy}$ at $60 \mu \mathrm{m}$, and $0.38 \mathrm{Jy}$ at $25 \mu \mathrm{m}$ for sources detected at both wavelengths, so that the only incompleteness in this sample occurs for the coldest sources, i.e., those with $0.2<f(25 \mu \mathrm{m}) / f(60 \mu \mathrm{m})<0.25$ and $f(60 \mu \mathrm{m})<1.9 \mathrm{Jy}$. From the distribution of $25 \mu \mathrm{m} / 60 \mu \mathrm{m}$ flux ratio for sources with $f(60 \mu \mathrm{m})>2.0 \mathrm{Jy}$, this incompleteness is not significant. Warm galaxies represent a small fraction of the galaxies in the IRAS database; simply scaling the BGS to the flux limit and area covered by the search for warm galaxies would have given $\sim 1800$ extragalactic sources detected at $60 \mu \mathrm{m}$. Thus the 90 sources in the warm galaxy survey represent $\sim 4 \%$ of the $60 \mu \mathrm{m}$ sources in the same area, very close to the $5 \%$ of the sources found in the BGS to meet the selection criteria of the warm galaxy survey and consistent with the fraction of warm galaxies found in the IRAS data by de Grijp, Miley, and Lub (1987). Thus we conclude that the galaxies selected as "warm" from the IRAS data do not represent a new class of objects previously excluded from samples selected at $60 \mu \mathrm{m}$.

\section{OBSERVATIONS}

Of the 90 sources in the warm galaxy sample, 15 had not been previously cataloged, while 22 did not have published redshifts. Of the objects without published redshifts, 11, preferentially chosen to be the faintest optical objects in the sample, were observed with the double spectrograph (Oke and Gunn 1982) on the Palomar $5 \mathrm{~m}$ telescope using the same configuration as described in Paper I. Including these observations, 12 objects in the warm sample were determined to be ultraluminous, i.e., $L \geq 10^{12} L_{\odot}$. Of the remaining galaxies in the warm sample without redshifts, all are sufficiently bright that the relation between infrared and optical luminosities for infrared selected galaxies (Paper II; Feigelson, Isobe, and Weedman 1987) suggests none will be high luminosity systems.

Optical and near infrared continuum measurements were made for all 12 ultraluminous objects in the warm sample. Photometric images in standard filters $(B, g, r, i)$ were obtained using the CCD camera on the Palomar $1.5 \mathrm{~m}$ telescope. Deep optical images of each of the ultraluminous objects were 


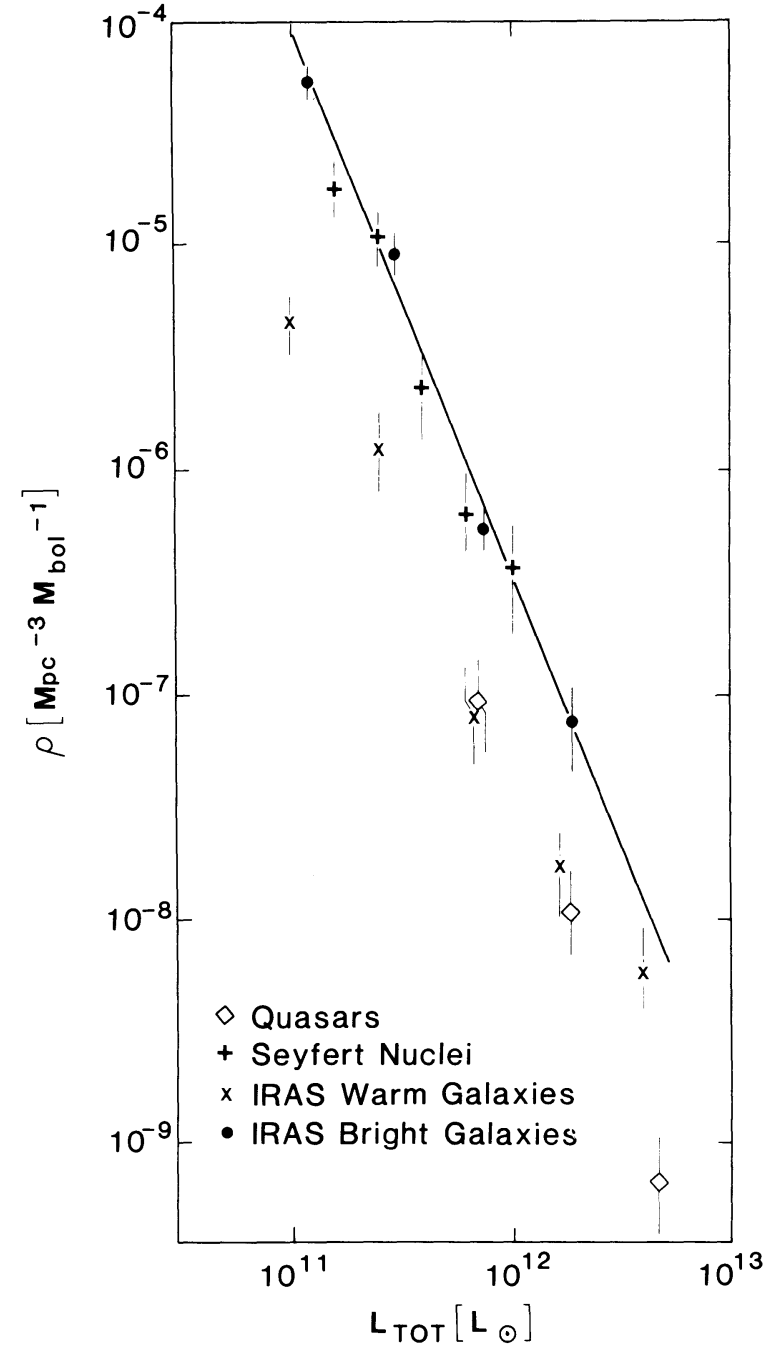

FIG. 1. - The luminosity functions of a variety of classes of extragalactic sources, normalized to the same Hubble constant $\left(H_{0}=75 \mathrm{~km} \mathrm{~s}^{-1} \mathrm{Mpc}^{-1}\right)$ and plotted in units of bolometric luminosity. The solid line represents the best fit to the IRAS Bright Galaxy ( filled circles) luminosity function (Paper II) The crosses represent the space density derived for the "warm "IRAS-detected galaxies. The diamonds represent optically selected quasars taken from Schmidt and Green (1983), and plus signs represent the optically selected Seyfert galaxies taken from Huchra (1977).

obtained by co-adding multiple exposures of each object taken at $6500 \AA$ (Gunn $r$ ). Near-infrared measurements in the $J(1.3$ $\mu \mathrm{m}), H(1.65 \mu \mathrm{m}), K(2.2 \mu \mathrm{m})$, and $L^{\prime}(3.7 \mu \mathrm{m})$ bands were made using a $10^{\prime \prime}$ diameter beam with the Palomar $5 \mathrm{~m}$ telescope. Measurements were also made at $N(10.1 \mu \mathrm{m})$ with a 4.6 beam using the $5 \mathrm{~m}$ telescope.

\section{LUMINOSITY FUNCTION}

The warm galaxies represent a subset of infrared bright galaxies having significantly greater luminosity than samples, such as the BGS, that are selected strictly on the basis of $60 \mu \mathrm{m}$ flux density. Excluding the galaxies without redshifts, the median infrared luminosity from $8 \mu \mathrm{m}$ to $1000 \mu \mathrm{m}$ (computed using the prescription outlined by Perault et al. 1988) of the warm sample is $\sim 6 \times 10^{10} L_{\odot}$ compared to $\sim 2.5 \times 10^{10} L_{\odot}$ for the BGS.

The space density versus infrared luminosity is plotted for the warm galaxies in Figure 1 for bolometric luminosities greater than $10^{11} L_{\odot}$. Also plotted are the space densities of the BGS, Seyfert galaxies, and quasars (see Paper II for the details of the bolometric corrections for the Seyferts and quasars). As in Paper II, the space density for the warm galaxies was calculated using the $1 / V_{m}$ technique (Schmidt 1968; Felten 1976). The warm galaxies have a significantly lower space density than that derived for the BGS for all bins included in both samples. At the higher luminosities, the space density of the warm galaxies represents a substantially higher fraction of infrared bright galaxies than the overall percentage. In the highest luminosity bins where there is overlap, the warm galaxies represent $15 \%-30 \%$ of the total of infrared luminous galaxies, consistent with the finding of Paper I that $3 / 10$ of the ultraluminous galaxies in the BGS meet the color criterion for inclusion in the warm galaxy sample.

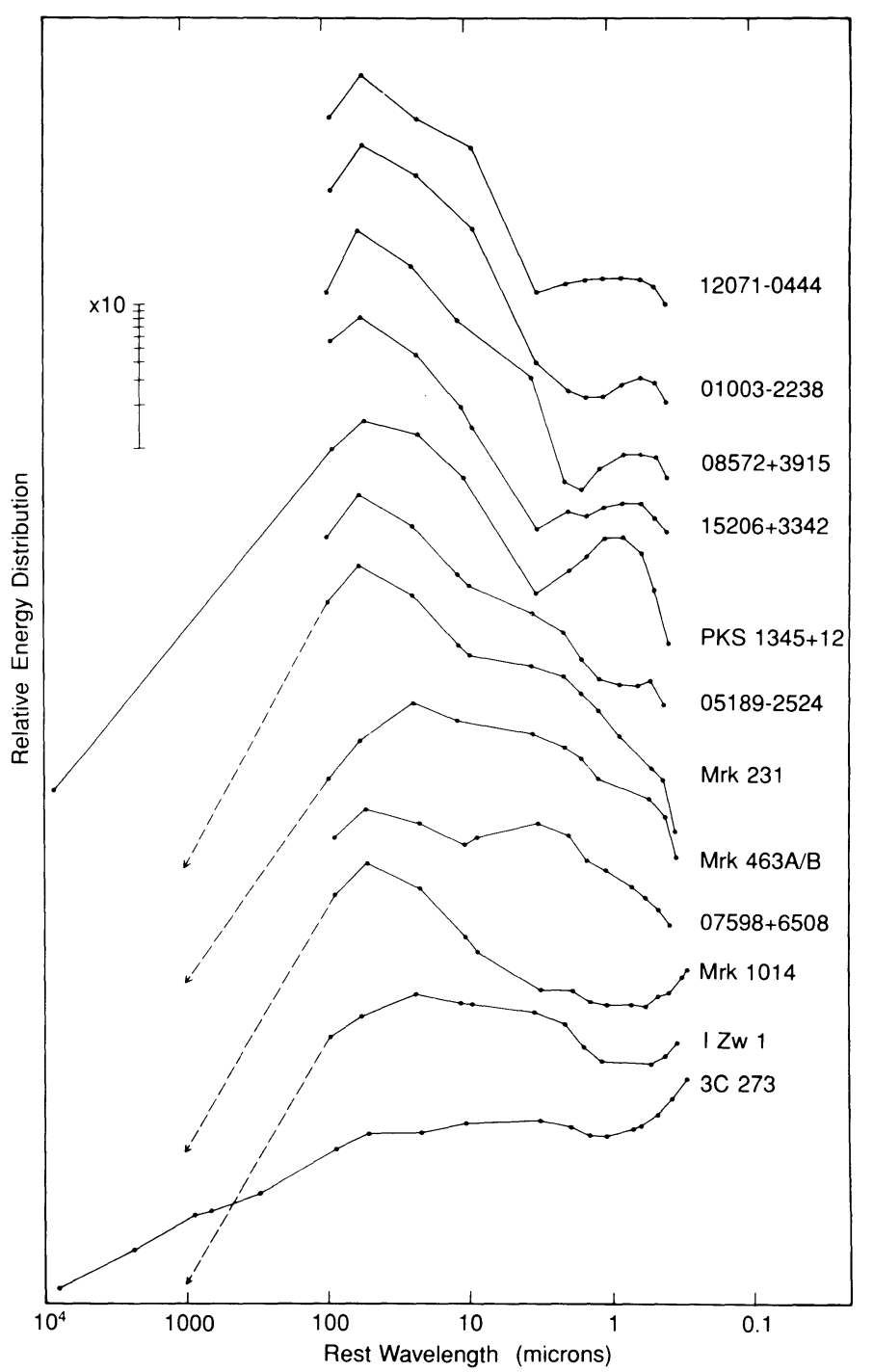

Fig. 2.-Spectral energy distributions for ultraluminous galaxies in the IRAS warm galaxy sample. The data from $0.44 \mu \mathrm{m}$ to $100 \mu \mathrm{m}$ are from this work. Data at $\lambda<0.4 \mu \mathrm{m}$ are from Neugebauer et al. $(1979,1986)$ or VéronCetty and Véron (1985). Data at $\lambda>900 \mu \mathrm{m}$ are from Kühr et al. (1981) and Kellerman et al. (1987). Dashed lines indicate an extrapolation beyond the figure boundary to the data point at $\lambda=6 \mathrm{~cm}$. 
V. PROPERTIES OF ULTRALUMINOUS OBJECTS IN THE WARM IRAS SAMPLE

The 12 objects in the warm sample with bolometric luminosities greater than $10^{12} L_{\odot}$ are listed in Table 1 . These objects meet the minimum bolometric luminosity criteria for quasars derived by adopting the criterion $M_{B}<-23.5$ (Schmidt and Green 1983) and integrating the luminosity over the wavelength range $0.01-100 \mu \mathrm{m}$ (Paper II). Three of the 12 objects have previously been reported as ultraluminous galaxies in the BGS (Paper I). The spectral classification of each of the ultraluminous warm objects is summarized in Table 1 . All of these objects are Seyferts, split equally between Seyfert 1 and Seyfert 2 galaxies.

The near-infrared and optical photometry from Palomar has been combined with the IRAS data to produce energy distributions for each object. The dominance of the infrared emission in the IRAS bands can easily be seen in Figure 2; the fraction of total luminosity contributed by emission at $\lambda \geq 8 \mu \mathrm{m}$ ranges from $\sim 90 \%$ for objects like IRAS $12071-0444$ (similar to the majority of the ultraluminous BGS objects) to approximately $50 \%$ for IRAS $07598+6508$. While the BGS contained no objects with strong UV excess, the warm ultraluminous sample contains a few such objects (e.g., Mrk 1014, I Zw 1, 3C 273). In general the infrared energy distributions of the ultraluminous warm objects are flatter than those for the BGS galaxies.

Nearly all of the objects in Table 1 have infrared luminosities greater than $10^{12} L_{\odot}$, and except for the radio-loud quasar 3C 273 (and possibly I $\mathrm{Zw} \mathrm{1)}$, the infrared luminosity dominates the energy output in every case. Neugebauer et al. (1979) conclude that the far-infrared emission in 3C 273 is dominated by nonthermal synchrotron emission, while in the other objects the marked peak in the distribution of $v f_{v}$ in the infrared suggests that the infrared emission is dominated by thermal emission from dust.

The strong peak in the energy distribution near $60 \mu \mathrm{m}$ provides evidence for large amounts of moderately cool dust $\left(T_{\text {dust }} \approx 45-55 \mathrm{~K}\right)$ in the majority of the ultraluminous "warm " objects, similar to the far-infrared distributions of the ultraluminous galaxies in the BGS. In addition, the warm objects also appear to have substantial emission in the $12 \mu \mathrm{m}$ and $25 \mu \mathrm{m}$
IRAS bands suggesting the presence of a significant component of hotter dust $\left(T_{\text {dust }}=100-200 \mathrm{~K}\right)$, similar to what is found for infrared Seyfert galaxies (Miley et al. 1984; Miley, Neugebauer, and Soifer 1985; de Grijp, Miley, and Lub 1987). There also appears to be substantial emission at 3-10 $\mu \mathrm{m}$ which could be due to even hotter dust emission $\left(T_{\text {dust }}=\right.$ $300-500 \mathrm{~K}$ ) or, possibly, to a nonthermal component directly associated with a Seyfert nucleus.

Deep CCD images of all 12 objects are shown in contour form in Figure 3. Morphologically eight of the 12 galaxies show clear evidence of interaction (double nuclei, tidal tails), one shows evidence of a distorted disk, while two are principally stellar. The remaining object, 3C 273, exhibits nebulosity on the $1.5 \mathrm{~m}$ image (see also Tyson, Baum, and Kreidl 1982) and has at least one prominent jet. We feel that the finding that the majority of the warm objects are strongly interacting galaxy systems supports the picture presented in Paper I whereby ultraluminous infrared galaxies and quasars are two phases in the evolution of strongly interacting, gas-rich spiral galaxies. At least 16 of the 19 ultraluminous objects in the combined BGS and warm object surveys are interacting galaxies.

In Paper I it was argued that molecular clouds could be funneled into a merger nucleus during the strong interaction/ merger of two gas-rich spiral galaxies where they could provide fuel for both an intense starburst and an active nucleus. Evidence for enormous gas supplies was provided by the detection of $\mathrm{CO}(1 \rightarrow 0)$ emission from five of the 10 ultraluminous BGS objects, including Mrk 231 and IRAS 05189-2524 that are also in the ultraluminous warm sample. Further evidence that large amounts of neutral gas are present in the majority of ultraluminous warm objects as well is provided by the detection of $\mathrm{CO}(1 \rightarrow 0)$ emission from Mrk 1014 and IRAS $15206+3342$ (Sanders, Scoville, and Soifer 1988), and by the detection of $\mathrm{H}_{\mathrm{I}}$ emission in I Zw 1 (Condon, Hutchings, and Gower 1985).

We believe the new data for the ultraluminous warm objects presented here support the picture for an intimate connection between ultraluminous infrared galaxies and quasars. The objects in Table 1 span many of the well-known classes of active galactic nuclei (AGN), so the distinction between ultra-

TABLE 1

Ultraluminous Galaxies in the IR $A S$ Warm Galaxy Sample

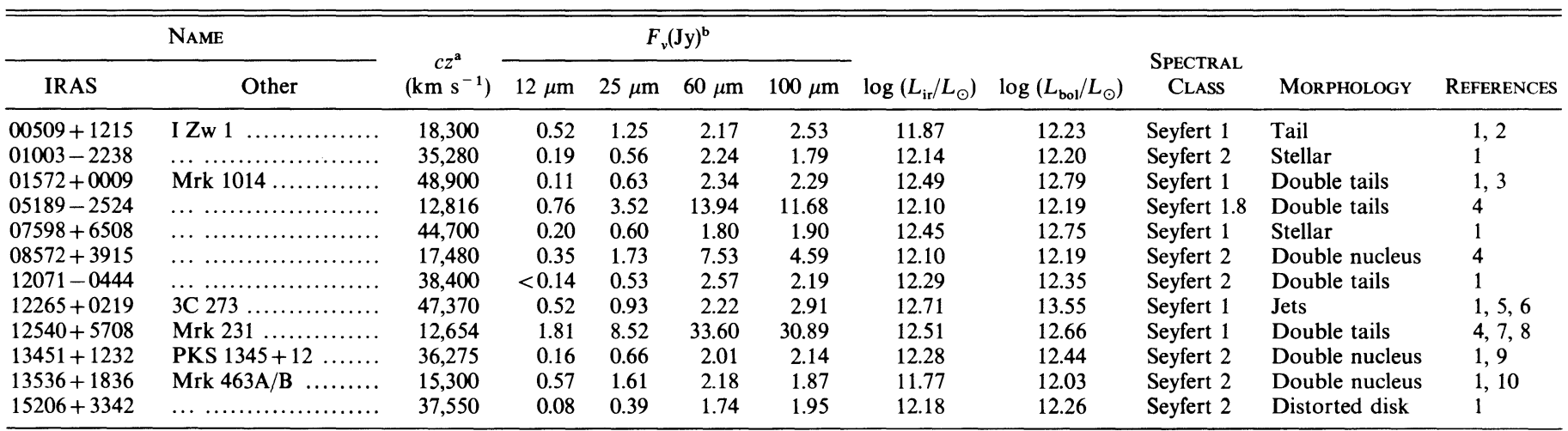

NoTE.-Objects in the IRAS warm galaxy sample with $L_{\mathrm{bol}}>10^{12} L_{\odot}$.

a Mean heliocentric redshift. In the case of Mrk 1014, IRAS 05189-2524, Mrk 231, and IRAS $15206+3342$, the redshift is determined from CO( $J=1 \rightarrow 0)$ data and has an uncertainty of $\pm 20 \mathrm{~km} \mathrm{~s}^{-1}$. In all other cases the redshift is taken from optical data and has an uncertainty of $\pm 100 \mathrm{~km} \mathrm{~s}^{-1}$.

b IRAS flux densities from co-added survey scans.

REFERENCES.-11) This work; (2) Phillips 1976; (3) MacKenty and Stokton 1984; (4) Sanders et al. 1988; (5) Tyson, Baum, and Kreidl 1982; (6) Schmidt 1963; (7) Arakelyan et al. 1971;(8) Sanders et al. 1987; (9) Grandi 1977;(10) Gilmore and Shaw 1986; (11) Petrosyan, Saakyan, and Khachikyan 1979. 

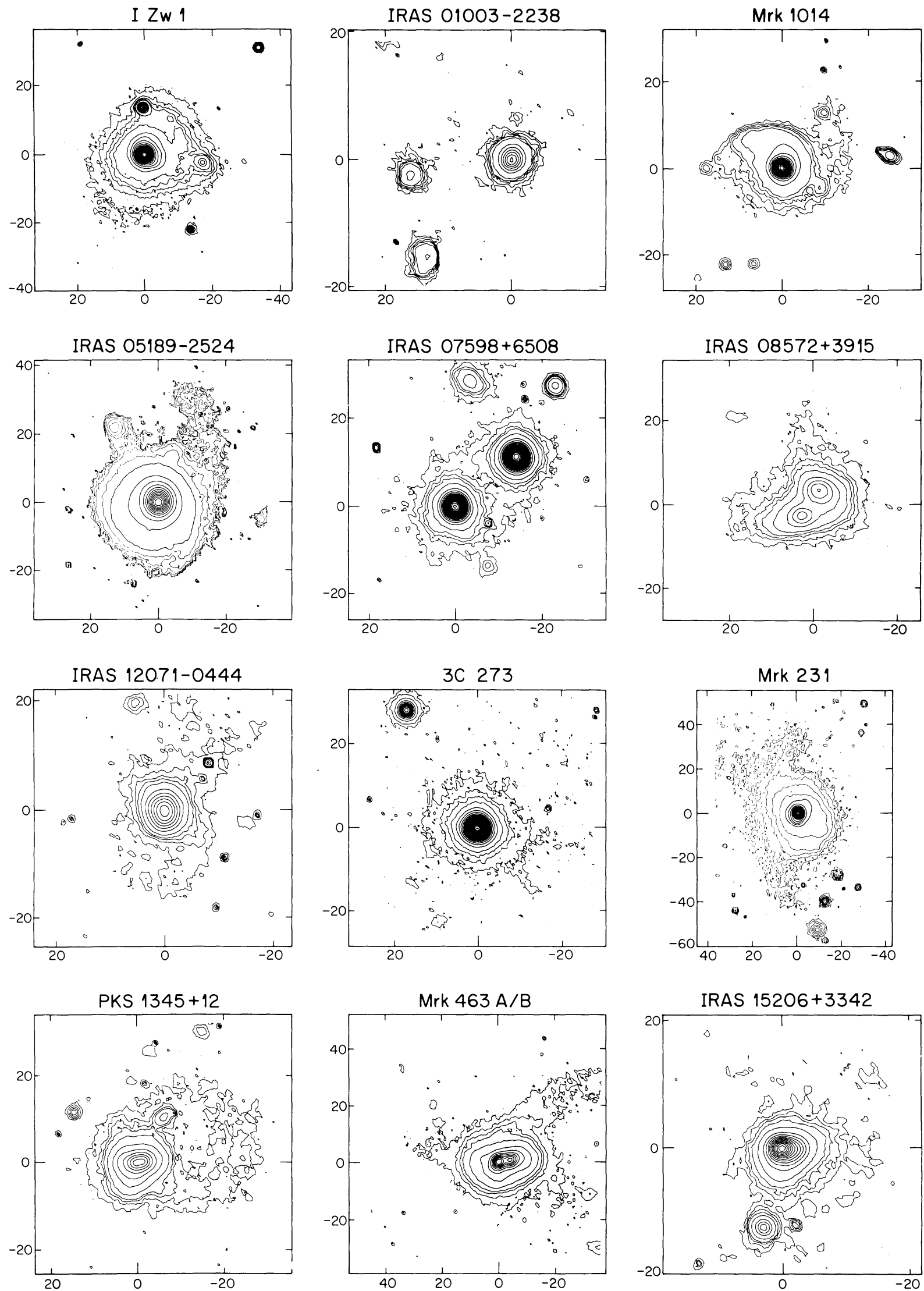

FIG. 3.-Contour maps from CCD images at $6500 \AA$ (Gunn $r$ ) of the 12 ultraluminous infrared galaxies from the IRAS warm galaxy survey. The images were obtained with the Palomar $1.5 \mathrm{~m}$ telescope. The lowest four contours are linear to emphasize faint large-scale structure, while the higher contours are logarithmic. The axes are labeled in arcseconds offset from the IRAS source. The companion object just to the northwest of IRAS $07598+6508$ is a star. 
luminous infrared, radio, or UV-loud AGN appears to be diminishing. We suggest that such distinctions are predominantly related to the interstellar medium surrounding the luminosity source and the viewing angle through which the nucleus is seen.

We also consider that the optical images of the infraredselected sample of objects in Table 1 strengthen the picture from Paper I that the ultraluminous infrared galaxies evolve into the classical quasars. Most of the ultraluminous warm objects contain luminous optical nuclei, so that the central powerhouse has apparently begun to break through the surrounding cloud of dust to reveal itself visibly. Qualitatively, the progression is from the objects with the greatest relative infrared luminosity being the most recognizable as galaxies to those with the least relative infrared luminosity having the brightest optical nucleus. The warm galaxy sample displays the transition from the cooler ultraluminous BGS galaxies to optical quasars by providing a significant sample of intermediate objects.

We thank our night assistants at Palomar, Juan Carasco and Skip Staples, for assistance in obtaining the observations. G. N., D. B. S., and B. T. S. were supported in part by the $I R A S$ extended mission program. Ground-based astronomy at Caltech is supported by a grant from the NSF. The Palomar $1.5 \mathrm{~m}$ telescope is operated jointly by the California Institute of Technology and the Carnegie Institution of Washington.

\section{REFERENCES}

Arakelyan, M. A., Dibai, É. A., Esipov, V. F., and Markaryan, B. E. 1971, Astrofizika, 7, 177.

Condon, J. J., Hutchings, J. B., and Gower, A. C. 1985, A.J., 90, 1642.

de Grijp, M. H. K., Miley, G. K., and Lub, J. 1987, preprint.

Felten, J. E. 1976, Ap. J., 207, 700 .

Feigelson, E. D., Isobe, T., and Weedman, D. 1987, Ap. J. (Letters), 319, L51.

Gilmore, G., and Shaw, M. 1986, Nature, 321, 750 .

Grandi, S. A. 1977, Ap. J., 215, 446.

Huchra, J. 1977, Ap. J. Suppl., 35, 171.

IRAS Catalogs and Atlases: Point Source Catalog. 1985, (Washington, DC: US Government Printing Office) (PSC)

Kellerman, K., et al. 1987, preprint.

Kühr, H., Witzel, A., Pauliny-Toth, I. I. K., and Nauber, U. 1981, Astr. Ap. Suppl., 45, 367.

Low, F. J., Huchra, J., Kleinman, S. G., and Cutri, R. M. 1988, Ap. J. (Letters), 327, L41.

MacKenty, J. W., and Stockton, A. 1984, Ap. J., 283, 64.

Miley, G., Neugebauer, G., Clegg, P. E., Harris, S., Rowan-Robinson, M., Soifer, B. T., and Young, E. 1984, Ap. J. (Letters), 278, L79.

Miley, G., Neugebauer, G., and Soifer, B. T. 1985, Ap. J. (Letters), 293, L11.

Neugebauer, G., Miley, G. K., Soifer, B. T., and Clegg, P. E. 1986, Ap. J., 308, 815.

Neugebauer, G., Oke, J. B., Becklin, E. E., and Matthews, K. 1979, Ap. J., 230, 79.

Oke, J. B., and Gunn, J. E. 1982, Pub. A.S.P., 94, 586.

Perault, M., Boulanger, F., Falgarone, E., and Puget, J. L. 1988, Astr. Ap., in press.

Petrosyan, A. R., Saakyan, K. A., and Khachikyan, E. E. 1979, Astrofizika, 15, 209.

Phillips, M. M. 1976, Ap. J., 208, 37.

Sanders, D. B., Scoville, N. Z., and Soifer, B. T. 1988, in preparation.

Sanders, D. B., Soifer, B. T., Elias, J. H., Madore, B. F., Matthews, K., Neugebauer, G., and Scoville, N. Z. 1988, Ap. J., 325, 74 (Paper I).

Sanders, D. B., Young, J. S., Scoville, N. Z., Soifer, B. T., and Danielson, G. E. 1987, Ap. J. (Letters), 312, L5. Schmidt, M. 1963, Nature, 197, 1040.

. 1968, Ap. J., 151, 393

Schmidt, M., and Green, R. F. 1983, Ap. J., 269, 352

Soifer, B. T., Sanders, D. B., Madore, B. F., Neugebauer, G., Danielson, G. E., Elias, J. H., Lonsdale, C. J., and Rice, W. L. 1987, Ap. J., 320, 238 (Paper II). Tyson, J. A., Baum, W. A., and Kreidl, T. 1982, Ap. J. (Letters), 257, L1.

Vader, J. P., and Simon, M. 1987, Nature, 327, 304.

Véron-Cetty, M.-P., and Véron, P. 1985, ESO Sci. Rept., No. 4.

\section{J. H. ELIAS: CTIO, Casilla 603, La Serena, Chile}

K. Matthews, G. Neugebauer, D. B. Sanders, and B. T. Soifer: Downs Lab, 320-47, California Institute of Technology, Pasadena, CA 91125 